Leader Humility and Team Performance: Exploring the Mechanisms of Team PsyCap and Task Allocation Effectiveness

\author{
Arménio Rego \\ Católica Porto Business School, Porto, Portugal \\ and
}

Business Research Unit (UNIDE-IUL), Instituto Universitário de Lisboa, Lisboa, Portugal

Bradley Owens

Marriott School of Management

Brigham Young University

Kai Chi (Sam) Yam

Business School

National University of Singapore

Dustin Bluhm

College of Business

University of Colorado Colorado Springs

Miguel Pina e Cunha

Nova School of Business and Economics

Universidade Nova de Lisboa, Portugal

Anthony Silard

California State University, San Bernardino

Lurdes Gonçalves

Universidade de Aveiro, Aveiro, Portugal

Mafalda Martins

Universidade de Aveiro, Aveiro, Portugal 


\author{
Ace Volkmann Simpson \\ UTS Business School \\ University of Technology, Sydney, Australia \\ Wenxing Liu \\ School of Business Administration \\ Zhongnan University of Economics and Law, Wuhan, China
}

Note: First, second, and third authors contributed equally to this project.

Acknowledgements: We thank Action Editor Bianca Beersma and two anonymous reviewers for their constructive comments throughout the review process. The authors are grateful to Luthans, Avolio, and Avey for having given us the permission to use the short version of PCQ. The authors are also very grateful to Susana Leal, Ana Ventura, and Andreia Vitória for their help in collecting data. This work was funded by National Funds through FCT - Fundação para a Ciência e Tecnologia under the project Ref. UID/ECO/00124/2013; by POR Lisboa under the project LISBOA-01-0145-FEDER007722; by a grant from the Templeton Foundation- Developing Humility in Leadership (60622); and by a fellowship from the Wheatley Institution at Brigham Young University. Wengxing Liu also wishes to thank The National Science Foundation of China: No. 71502175

Corresponding author: Wenxing Liu, School of Business Administration, Zhongnan University of Economics and Law, 182\# Nanhu Avenue, East Lake Hightech Development Zone, Wuhan 430073, P.R. China

Email:1wx856@znufe.edu.cn 


\title{
Leader Humility and Team Performance: Exploring the Mechanisms of Team PsyCap and Task Allocation Effectiveness
}

\begin{abstract}
Although there is a growing interest toward the topic of leader humility, extant research has largely failed to consider the underlying mechanisms through which leader humility influences team outcomes. In this research, we integrate the emerging literature of leader humility and social information processing theory to theorize how leader humility facilitates the development of collective team psychological capital, leading to higher team task allocation effectiveness and team performance. While Owens and Hekman (2016) suggest that leader humility has homogenous effects on followers, we propose a potential heterogeneous effect based on the complementarity literature (e.g., Tiedens, Unzueta, \& Young, 2007) and the principle of equifinality (leaders may influence team outcomes through multiple pathways; Morgeson, DeRue, \& Karam, 2010). In three studies conducted in China, Singapore, and Portugal, including an experiment, a multisource field study, and a three-wave multisource field study, we find support for our hypotheses that leader humility enhances team performance serially through increased team psychological capital and team task allocation effectiveness. We discuss the theoretical implications of our work to the leader humility, psychological capital, and team effectiveness literatures; and offer suggestions for future research.
\end{abstract}

Keywords: leader humility; team psychological capital; team task allocation effectiveness; team effectiveness 


\section{INTRODUCTION}

"Humility is the solid foundation of all virtues."

- Confucius

"If leadership has a secret sauce, it may well be humility"

—The Economist (2013)

Humility has historically been considered a foundation for virtues and character strengths (see Grenberg, 2005; Krieger, 2002; Morris, Brotheridge, \& Urbanski, 2005). While classical ideas about humility focus on the intrapersonal benefits of humility in developing other positive personal qualities (e.g., Kesebir, 2014; Roberts \& Wood, 2003; Tangney, 2009), contemporary studies of humility in organizations focus on the relational influence of humility and its social impact on others (e.g., Ou, Waldman, \& Peterson, 2015; Owens, Johnson, \& Mitchell, 2013). Defined as an interpersonal characteristic that emerges in social contexts that connotes (a) a willingness to view oneself accurately, (b) an appreciation of others' strengths and contributions, and (c) teachability, or openness to new ideas and feedback (Owens et al., 2013), initial qualitative and quantitative evidence suggests that leader humility may help foster adaptive strengths within the teams they lead (Owens \& Hekman, 2012), which ultimately enhances team performance (Owens \& Hekman, 2016).

But the impact of leader humility on the development of character strengths in others has received little theoretical elaboration and scant empirical attention. Understanding this potential influence will further illuminate why leader humility has positive impact on the teams they lead, and more generally, shed light on the role and relevance of the historic virtue of humility in our contemporary organizations. Thus, drawing from social information processing theory (SIP; Salancik \& Pfeffer, 1978) and the emerging leader humility literature (Oc, Basshur, Daniels, Greguras, \& Diefendorff, 2015; Ou,Tsui, Kinicki, Waldman, Xiao, \& Song, 2014; Owens \& Hekman, 2012), the 
purpose of this research is to empirically examine how leader humility fosters collective strengths in teams, captured by team psychological capital (PsyCap), leading to higher team effectiveness.

In this paper, we define PsyCap as a positive, developmental state comprising the strengths of hope, efficacy, resilience, and optimism (Luthans, Youssef-Morgan, \& Avolio, 2015). As traditional leadership approaches have been "criticized for failing to fully appreciate and model the dynamism and complexities of team leadership" (Burke et al., 2006, p. 302), scholars have recently begun to explore the influence of the nontraditional leadership approach of leader humility on team processes and team performance (Ou et al., 2014, 2015; Owens \& Hekman, 2016). Recent empirical research on the positive benefits of leader humility on teams has documented that humble leadership is associated with: lowered pay disparity, higher team integration (i.e., team members' willingness to adopt collaborative behaviors, share information, make joint decisions, and develop a shared vision; Ou et al., 2014) and ambidextrous strategic orientation (i.e., "exploiting existing competencies as well as exploring new opportunities with equal dexterity"; Lubatkin, Simsek, Ling, \& Veiga, 2006, p. 647; see also $\mathrm{Ou}$ et al., 2015); positive team interaction patterns reflecting the dimensions of humility (collective humility); and team promotion regulatory focus (Owens \& Hekman, 2016). While these capture positive team-level mechanisms enabled by leader humility (i.e., impacting relative pay, strategic orientation, and behavioral mimicry), they do not address how or whether expressed humility may be a catalyst of strengths in others, captured by team PsyCap.

At the team level, team PsyCap represents the agreement among team members in regard to the team's shared PsyCap perception (Dawkins, Martin, Scott, \& Sanderson, 2015). Team PsyCap is a team property "produced through interactions 
directly relating to the team (e.g., team-related goal planning) and is thereby distinct from the individual PsyCap perceptions that team members hold about themselves" (Dawkins et al., 2015, p. 936). We propose that leaders who show humility develop team PsyCap because, in line with SIP theory, humble leader behaviors represent powerful social cues that shape the perceptions of the teams they lead, which facilitate the development of the strengths comprised in PsyCap. Specifically, leader humility has been qualitatively documented to facilitate adaptive responses to mistakes and weaknesses, experiment with new ways of accomplishing the work, create a sense of validation of strengths, and adopt a positive, growth-based, developmental paradigm about organizational life (Owens \& Hekman, 2012), which corresponds with the dimensions of PsyCap.

We further theorize that team PsyCap in turn helps teams maximize their human capital by mediating the positive effects of leader humility on team task allocation effectiveness and team performance. As suggested by qualitative (Owens \& Hekman, 2012) and quantitative evidence (Owens \& Hekman, 2016), task allocation effectiveness is a key team effectiveness process that might be facilitated by leader humility. In our theoretical model, we explain why the collective properties associated with team PsyCap mediate the effects of leader humility on the process of pairing team members with team tasks according to members' individual strengths.

Reflecting the components of team process modeling defined by Marks, Mathieu, and Zaccaro (2001), we organize our theoretical model by proposing leader humility as an important team input that shapes the team emergent state of team PsyCap, leading to the team process of team task allocation effectiveness, which fosters the outcome of team performance (see Ilgen, Hollenbeck, Johnson, \& Jundt, 2005), 
which is the most commonly studied criterion reflecting team effectiveness (Burke et al., 2006; Ilgen et al., 2005).

By examining the serial underlying mechanisms (i.e., team PsyCap and team allocation effectiveness) of the effects of leader humility on team performance, this research makes four contributions to the literature. First, we seek to build off of the recently documented positive effects of leader humility on teams (i.e., team's strategic orientation, interaction patterns, and relative pay disparity; Ou et al., 2015; Owens \& Hekman, 2016). To build off of, yet differentiate our work from Owens \& Hekman (2016), we propose a potential heterogeneous effect based on the complementarity literature (Grant, Gino, \& Hoffman, 2011; Tiedens, Unzueta, \& Young, 2007) and the principle of equifinality wherein leaders may influence team outcomes through multiple pathways (Morgeson, DeRue, \& Karam, 2010).

Second, this research contributes to the growing leader humility literature as it is the first to examine how leader humility influences the development of team deep-level strengths, in the form of team PsyCap. More critically, this research examines the mechanisms that drive the positive effects of leader humility. Doing so allows us to create a better understanding of leader humility, because examining underlying mechanisms is a critical component of theory building that helps scholars identify why a process occurs (see Colquitt \& Zapata-Phelan, 2007).

Third, this study furthers our understanding of how the nontraditional leadership approach of leader humility fosters team effectiveness across three different cultures (Portugal, Singapore, China; Hofstede, 2016a, 2016b, 2016c; Hofstede, Hofstede, \& Zinkov, 2010; Wang, Wang, Ruona, \& Rojewski, 2005), in which humility in leaders is valued differently (Mittal \& Dorfman, 2012; Morris et al., 2005; Oc et al., 2015; Ou et al., 2014; Vera \& Rodriguez-Lopez, 2004). 
Fourth, this research contributes to research on team PsyCap as it reveals a novel antecedent (i.e., leader humility) and illuminates the connection between team PsyCap and team performance through task allocation effectiveness. As PsyCap reflects a collection of positive states that can be developed, more fully understanding the leadership antecedents of team PsyCap would enhance our theoretical understanding of this positive, adaptive construct and lend practical insight about fostering PsyCap within organizations. In other words, the current research uncovers how teams with higher PsyCap collectively shape a better work experience by being aware of and maximizing the use of the unique expertise or skills diffused throughout the team. Table 1 summarizes our theoretical model and includes the construct type, aggregation logic, and definition of each focal variable.

Insert Table 1 about here

\section{THEORETICAL BACKGROUND}

\section{Leader Humility}

The study of virtues in general, and humility specifically, has seen rapid expansion with the rise of Positive Psychology and Positive Organizational Scholarship (Cameron \& Spreitzer, 2012; Cameron \& Winn, 2012; Peterson \& Seligman, 2004; Van Dierendonck, 2011). Reflecting the definition given above, humility influences the way we see ourselves (more objectively), others (more appreciatively), and new information or ideas (more openly). Since the development of a validated measure of leader humility (Owens et al., 2013), a number of scholars have found that leader humility is associated with a range of positive follower outcomes, such as increased promotion focus, leader 
trust, job satisfaction, engagement, retention, and job performance (Basford, Offermann, \& Behrend, 2014; Ou et al., 2014; Owens et al., 2013).

The word humility comes from the Latin humus, meaning "soil," and humi, meaning "on the ground" (Owens \& Hekman, 2012) and has been called the fertile soil from which all other virtues grow (Watts, 2011; see also Awtry, 2007, and Grenberg, 2005). Rather than being like static traits we possess, classical (i.e., Aristotle, Plato) and contemporary philosophers view virtues as being like moral skills that can be developed, or moral muscles that can be grown (see Bloomfield, 2000; Stichter, 2007, 2011). It was Aristotle (1999, p. 20) who argued that "moral virtue comes about as a result of habit, whence also its name (ethike) is one that is formed by a slight variation from the word ethos (habit)." Though philosophers have extolled humility as being foundational to developing virtue or character strength and extant qualitative evidence suggests that followers see leader humility as modeling how to grow and that leader humility helps followers embrace their own developmental journeys (Owens \& Hekman, 2012), however, there is very little empirical understanding of how leader humility actually influences deep-level follower development of character strengths.

Building off of this study, we theorize that leader humility can engender important strengths in the form of PsyCap within a team when the team is on the receiving end of humility enacted by a team leader in the workplace (see above arguments about potential heterogeneous effect based on the complementarity literature principle of equifinality). Specifically, we propose that as leaders admit mistakes, shine the spotlight on others' strengths, and seek to be taught by others, they give away power, producing a complementary "empowered" followership who feel an added level of PsyCap.

\section{Team PsyCap}


Individual PsyCap connotes a positive psychological state of development characterized by "(1) having confidence (efficacy) to take on and put in the necessary effort to succeed at challenging tasks; (2) making a positive attribution (optimism) about succeeding now and in the future; (3) persevering toward goals and, when necessary, redirecting paths to goals (hope) in order to succeed; and (4) when beset by problems and adversity, sustaining and bouncing back and even beyond (resilience) to attain success" (Luthans et al., 2015, p. 2). Conceptual (Luthans et al., 2015) and empirical (Avey et al., 2011) evidence suggests that commonalities and synergies among the four components allow considering PsyCap as a core, or a higher-order, construct. Although other positive psychological resources (e.g., gratitude, courage, creativity and wisdom) could be included in this study, the strengths of hope, efficacy, resilience, and optimism are those that, in the current "state of art," clearly satisfy the three most relevant POB (Positive Organizational Behavior) criteria: they can be measured, developed, and effectively managed for performance improvement in workplaces (Luthans et al., 2015). As a core construct, PsyCap represents “one's positive appraisal of circumstances and probability for success based on motivated effort and perseverance" (Luthans, Avolio, Avey, \& Norman, 2007, p. 550). Likewise, a recent meta-analysis revealed a significant and positive correlation between PsyCap and numerous desirable work attitudes and outcomes, including both subjective and objective measures of job performance (Avey et al., 2011).

Although PsyCap has been primarily studied at the individual level, an increasing number of studies also demonstrate that PsyCap develops at the collective/team level and that collective/team PsyCap predicts team performance (see Dawkins et al., 2015). The dimensions of PsyCap at the team level have been proposed to be isomorphic representations of individual level PsyCap (West, Patera, \& Carsten, 2009) and may be 
operationalized as a reference shift composition model (Chan, 1998; alternate conceptualizations of collective PsyCap in Dawkins et al., 2015). As a shared team property, team PsyCap originates in individual unit members" "experiences, attitudes, perceptions, values, cognitions, or behaviors and converge among group members as a function of . . socialization, social interaction, [and] leadership" (Kozlowski \& Klein, 2000, p. 19). When compared to individual PsyCap, the shared property of team PsyCap may reflect a more consistent sense of efficacy, optimism, hopefulness, and resilience because it is socially reinforced and less likely to fluctuate due to individual moods or self-evaluative cognitions. In addition, PsyCap on the team level represents more varied total resources that exist beyond an individual member that can be used to shore up against challenges or recover from bouts of failure. We link the development of shared PsyCap in a team with SIP theory (Salancik \& Pfeffer, 1978).

\section{Leader Humility and Team PsyCap}

Based on SIP theory (Salancik \& Pfeffer, 1978), we propose that leader humility is experienced and interpreted by individual followers as they engage in social interaction and make sense of consistent social cues given by their leader. SIP theory suggests that individuals make sense of and understand their work environments through processing social information, which in turn shapes their work attitudes and behaviors (Salancik \& Pfeffer, 1978). Leaders are vital sources of social information because of their high status and direct interactions with those they lead (e.g., Yaffe \& Kark, 2011). We propose that over time, the effect of the leader's modeling of humility will reinforce certain individual beliefs that will be shared by the team. Generally speaking, this connection is reasonable because PsyCap is developable, and leader humility legitimizes follower development (Owens \& Hekman, 2012), putting followers in a frame of mind that development is a positive, valid, and appropriate pursuit. 
However, looking more specifically at the dimensions of leader humility, the unique cues from the leader that reinforce or facilitate team PsyCap perceptions are as follows: First, as leaders validate and make unique team member strengths and contributions socially salient, these members are likely to feel an increased sense of confidence (self-efficacy) in their own abilities within the team context and to be aware of the unique strengths or expertise of their team members, leading to a heightened collective sense of efficacy. Second, as suggested by qualitative work, the humble leader behavior of admitting limits and mistakes is typically enacted in the context of describing some growth or developmental narrative (Owens \& Hekman, 2012). Rather than to self-deprecate, the leader's admission of his/her limits and mistakes has productive effects on team members, at both the individual and collective levels, and is meant to inspire growth or development in followers. This self-disclosure on the part of the leader suggests that mistakes, challenges, and setbacks can be overcome and can facilitate growth, which we propose will reinforce collective beliefs about the PsyCap dimensions of hope, optimism, and resilience. In addition, followers interpret leaders' admitting of mistakes as relief from the need to maintain a perfect persona or to selfenhance (Owens \& Hekman, 2012). This is important in terms of facilitating greater PsyCap, as self-enhancement or an inflated self-view has been linked to tendencies that appear to run contrary to the construct of PsyCap and its specific dimensions of hope, resilience, and optimism (see Colvin, Block, \& Funder, 1995).

Third, teachability reinforces openness to ongoing feedback about one's current course of action. As leaders model and sanction this behavior it will facilitate teams' ability to embrace the belief that continually evaluating and updating their progress and effectiveness will enable them to "identify, clarify, and pursue the way to success" and realize "alternative ways to reach [team] goals" (Luthans et al., 2007, p. 546). Thus, 
leader teachability enables teams to realize the way to success on an ongoing basis, which reflects one component of the hopefulness dimension of PsyCap.

In short, the three behavioral components of humility in team leaders allow team members, both individually and collectively, to have mastery experiences that lead to higher self-efficacy. Those behaviors also communicate confidence in the team's capacities, thus enriching the team's sense of agency and willpower (i.e., hope). With those actions, humble leaders make the team more adaptive and prepared to resiliently face risks, drawbacks, and failures (Lengnick-Hall, Beck, \& Lengnick-Hall, 2011). Those leader's actions also provide the team with more flexible perspectives about the future and lead the team to embrace future uncertainties with positive, welcoming, confident, and optimistic attitudes (Luthans \& Youssef, 2004; Luthans et al., 2015; Youssef \& Luthans, 2012). Considering the synergies between the four PsyCap dimensions mentioned above and that PsyCap develops at the team level through mechanisms discussed previously, it is possible that humble leaders nurture team PsyCap.

We further expect that these properties of team PsyCap, which we argue are facilitated by leader humility will in turn enhance team performance. A handful of empirical investigations suggest that PsyCap has a strong positive influence on individual job performance (see meta-analysis by Avey et al., 2011), and recent research suggests that this association is also observed at the team level (Clapp-Smith, Vogelgesang, \& Avey, 2009; Haar, Roche, \& Luthans, 2014; Heled, Somech, \& Waters, 2016; Mathe-Soulek, Scott-Halsell, Kim, \& Krawczyk, 2014; Vanno, Kaemkate, \& Wongwanich, 2015; West et al., 2009). PsyCap has been considered a source of competitive advantage beyond economic, human and social capital (Luthans et al., 2015; Luthans \& Youssef, 2004). Teams with higher PsyCap are more effective 
because they (a) set challenging goals and carry out the necessary efforts to succeed in pursuing such goals, (b) persevere in those goals' pursuit and generate different pathways to face problems and opportunities, (c) develop positive expectations about results and make positive attributions about succeeding now and in the future, and (d) sustain, bounce back, and even grow stronger when beset by setbacks, failures, and adversity (Avey et al., 2011; Dawkins et al., 2015; Luthans et al., 2015). In short, psychologically stronger teams are more likely to develop agentic behaviors, both individually and collectively, toward successfully accomplishing goals and tasks leading to better team performance. Together, we expect that team PsyCap will mediate the relationship between leader humility and team performance and posit the following hypotheses:

Hypothesis 1: Leader humility will be positively associated with team PsyCap.

Hypothesis 2: Leader humility will have an indirect relationship with team performance, via team PsyCap.

\section{Team Task Allocation Effectiveness}

Team task allocation effectiveness reflects demands-abilities fit within a team and the maximization of collective human capital. This variable is operationalized as an additive composition model (Chan, 1998) and represents a task work process (i.e., how the work gets done). In line with Marks and colleagues's (2001) taxonomy of team functioning, task allocation effectiveness reflects elements of both the transition and action stages as it captures team's evaluative efforts and planning activities to achieve greater effectiveness (i.e., more effectively aligning team tasks with member's capabilities and strengths) and captures behaviors that lead to goal accomplishment, respectively. Rather than focusing on general team skills common across all team members, aligning specific member skills to specific team tasks is proposed to be vital 
for effective team functioning (Marks, Mathieu, \& Zaccaro, 2001). We propose that teams with higher PsyCap will develop greater task allocation effectiveness. Specifically, teams with higher awareness of who is good at what on the team (i.e., efficacy) will be better able to make informed and effective decisions about which team member(s) should spearhead which team functions or tasks (Lewis, 2003; Moreland, 2006). In addition, the PsyCap dimensions of believing in trying different paths to achieve goals (hope) and making adaptive changes after episodes of failure (resilience), would make it more likely that teams will iteratively evaluate the pairings of team tasks with member strengths and make adaptations to form better fitting pairings (see Brandon \& Hollingshead, 2004). Over time, these adaptive and strengths-awareness tendencies will work toward tasks settling on team members according to their unique skills, strengths, and abilities, which is captured by task allocation effectiveness (see Owens \& Hekman 2012, 2016).

In addition, team PsyCap develops in part through mental model convergence (Mathieu, Heffner, Goodwin, Sales, \& Cannon-Bowers, 2000). One type of mental model convergence fostered by leader humility is a shared mental model of who possesses which types of expertise or skills (Owens \& Hekman, 2012, 2016), which influences the team's collective sense of efficacy. This collective sense of team efficacy, comprised in PsyCap, reflects a shared awareness of team capability and we suggest is formed in part by a humble leader's overt efforts to make the strengths of team members socially salient. The leader does this by drawing attention to or spotlighting the specific strengths, aptitudes, and contributions of those they lead, which enhances collective awareness of the team's diffused capabilities. In light of these advocacy and mental model arguments, we posit that team PsyCap is a mechanism through which leader humility fosters team task allocation effectiveness. 
As team members are allocated tasks that best match their skills and expertise, the team maximizes its human capital and, thus, enhances its likelihood of maximizing the team's output. One of the core organizing logics for teams is the synergy created when members coordinate efforts according to their unique strengths. This synergy enables performance beyond that which could be achieved by the sum of each individual's efforts. Hence, we argue that the positive influence of leader humility and team PsyCap on team performance will be achieved through team task-allocation effectiveness.

Hypothesis 3: Leader humility will have an indirect relationship with team performance, via team PsyCap and team task allocation effectiveness serially.

\section{RESEARCH OVERVIEW}

We carried out three studies to test our hypotheses (Table 1). In order to establish causal validity between leader humility and team PsyCap and to increase the crosscultural validity of our work, in our first study we examine Hypothesis 1 experimentally, specifically through a vignette-based leader humility experiment (Van Doorn, Heerdink, \& Van Kleef, 2012; Van Kleef, De Dreu, \& Manstead, 2004, 2006). Aguinis and Bradley (2014, p. 2) argued that such a methodology "enhances experimental realism and also allows researchers to manipulate and control independent variables, thereby simultaneously enhancing both internal and external validity." Study 1 was thus specifically designed to establish causal inferences and examine the effects of leader humility on team PsyCap (Hypothesis 1) across two cultures (Singapore and Portugal). In Study 2 we tested the first part of our serial indirect effect model (Hypotheses 1 and 2) using multisource data from organizations in Portugal. In Study 3, we tested the full serial indirect effect model (Hypothesis 3), replicating and extending Studies 1 and 2 using multiwave, multisource data from organizations in China. In the 
Portuguese and Chinese samples, all materials were originally written in English and subsequently translated into Portuguese and Chinese following established best practice of survey translation (Brislin, 1970).

\section{STUDY 1 METHODS}

\section{Participants and Procedure}

Our first sample consisted of 97 undergraduate students $\left(M_{\text {age }}=20.92,54.6 \%\right.$ male) from a business school in Singapore $(n=23$ teams). Our second sample consisted of 229 Portuguese students $\left(M_{\mathrm{age}}=26.07,64.2 \%\right.$ female $)$ recruited from two business schools in Portugal $(n=74$ teams). The majority of participants $(80.4 \%$, Singapore; $58.5 \%$, Portugal) indicated prior work experience, and this contributes to improve realism by increasing (a) the similarity between the experimental and the natural setting and (b) "the subjective experience of being personally immersed in the situation described in the vignette" (Aguinis \& Bradley, 2014, p. 11). With both samples, we conducted a between-subjects experiment manipulating leader humility. All participants have been working with teams of three to five students (Singaporean sample) or two to six students (Portuguese sample) from the beginning of the semester and were asked to complete this study toward the end of the semester. In other words, team members have been working with each other for over two months.

During the study, teams were randomly assigned to either a humble leader or control condition in which a transactional leader was presented. We used transactional leadership in the control condition (van Dierendonck, Stamn, Boersma, de Windt, \& Alkema, 2014) because transactional leadership is neutral in terms of humility. Moreover, manipulating non-humble leadership might introduce other unintended confounds such as increased perceptions of narcissistic leadership, which has been shown to be theoretically and empirically distinct from low leader humility (Ou et al., 
2014; Owens et al., 2013; Owens, Wallace, \& Waldman, 2015). All team members were presented with a short description of their direct supervisor and were first asked to read the scenario independently. Then, team members collectively discussed for five minutes how it would be to work with the leader. At the end of the discussion, all participants completed the team PsyCap scale independently.

\section{Manipulations and Measures}

Leader humility manipulation. We manipulated leader humility based on predominate conceptualizations of the construct within the existing literature (Owens et al., 2013; Owens \& Hekman, 2016). Specifically, we manipulated leader humility by emphasizing three major components of the construct discussed in the literaturewillingness to view oneself accurately, appreciation of others' strengths, and teachability (Owens et al., 2013).

In the humble leader condition, participants were told that their direct supervisor, John, is a supervisor very aware of his personal strengths and weaknesses; that John often solicits feedback and ideas from his employees; and that John shows appreciation for the unique contributions of others and often compliments others on their strengths and qualities. All of these humble leadership behaviors are taken directly from a wellestablished scale of leader humility (Owens et al., 2013) and similar manipulations of leader humility have been used in prior research (Owens \& Hekman, 2016). In the control condition, participants were provided with a scenario of a transactional leader. Participants were told that their direct supervisor, John, rewards good performance and takes actions to prevent bad performance. Importantly, in this scenario the leader does not display any qualities reminiscent of leader humility. Both scenarios are available in the Appendix. 
Team PsyCap. Immediately following the manipulations, participants completed the Psychological Capital Questionnaire-12 (or PCQ-12) (Avey et al., 2011), a shortened but validated version of the PCQ-24 [Insert Footnote 1 Here] (Luthans et al., 2015). Following past research, we adapted the items to the team level (West et al., 2009) using a referent-shift format (Chan, 1998). Sample items are: (1) "Team members feel confident contributing to discussions about the organization's strategy" (selfefficacy); (2) "If team members should find themselves in a jam at work, they could think of many ways to get out of it" (hope); (3) "Team members can get through difficult times at work because they have experienced difficulty before" (resilience); (4) "Team members are optimistic about what will happen to them in the future as it pertains to work" (optimism) $(1=$ "strongly disagree" to $6=$ "strongly agree"; Alphas $=$ .95 , Singaporean sample; .96, Portuguese sample). Because we did not theorize leader humility to have differentiated impact on these sub-factors of team PsyCap, an overall PsyCap score was used in all subsequent analyses.

Manipulation check. After completing the team PsyCap scale, participants were asked to respond to a one-item manipulation check: "I would characterize John as a humble leader" $(1=$ strongly disagree to 7 = strongly agree $)$.

Preliminary Analysis. To ensure that data aggregation is appropriate, we calculated ICC1, ICC2, and $r_{w g(J)}$ for the team PsyCap construct. Data aggregation is considered appropriate when ICC1 is non-zero (Bliese, 2000) and when ICC2 is higher than .70 (Kozlowski \& Klein, 2000). The ICC1s were .69 and .65, respectively, for the Singaporean and Portuguese samples. The ICC2s were .90 and .85, also respectively. The $r_{w g(J)}$ values for the Singaporean sample (average: .84; medium: .84; percentage of values higher than .70: 91\%) and the Portuguese sample $(.90 ; .93 ; 97 \%)$ represent 
“strong" inter-rater agreement (LeBreton \& Senter, 2008, p. 836). Considering these results, we proceeded to aggregate our data for team PsyCap.

\section{Results}

As expected, the manipulation check suggested that participants in the leader humility condition rated the leader as significantly more humble than participants in the control condition, for both the Singaporean $(M=5.65, S D=1.15$ versus $M=2.91, S D=$ $\left.1.50, \mathrm{t}_{(95)}=10.15, p<.01\right)$ and the Portuguese samples $(M=5.87, S D=1.11$ versus $M=$ 4.34, $\left.S D=1.71, \mathrm{t}_{(227)}=8.13, p<.01\right)$. These results provided support that our manipulation was effective in eliciting participants in imagining themselves working with a humble leader. The teams submitted to the leader humility condition $(\mathrm{n}=12$ teams, Singapore; $\mathrm{n}=35$, Portugal) rated the team PsyCap as significantly higher compared to the teams submitted to the control condition $(n=11$ teams, Singapore; $n=$ 39, Portugal), both in the Singaporean $(M=4.54, S D=.44$ versus $M=2.98, S D=.37$, $\left.\mathrm{t}_{(21)}=9.04, p<.01\right)$ and the Portuguese $(M=4.53, S D=.50$ versus $M=3.85, S D=$ $\left.1.02, \mathrm{t}_{(72)}=3.69, p<.01\right)$ samples. When both samples are combined, the main effect of leader humility on team PsyCap remained significant $(M=4.54, S D=.49$ versus $M=$ 3.64, $\left.S D=.98, \mathrm{t}_{(95)}=5.75, p<.01\right)$.

\section{Study 1 Discussion}

The findings suggest that, as hypothesized, leader humility influences team PsyCap. Specifically, leader humility influences the expectations team members have about PsyCap, not actual PsyCap. Although measuring expectations of PsyCap instead of actual PsyCap is a limitation of the study (other limitations below), the empirical pattern is consistent across both samples (and with evidence emerging from the next two studies), which reinforces the notion of leader humility as an important driver for team outcomes across cultures (Morris et al., 2005; Ou et al., 2015). Several limitations 
(beyond the one of measuring expectations of PsyCap instead of actual PsyCap) are however worth mentioning.

Although the study supports the causal direction of our proposed model, it was carried out through scenarios, instead of with observed real behaviors. Only the link between leader humility and team PsyCap (H1) was studied. It is possible that our findings represent, at least partially, the effects of participants' implicit theory of leadership (Junker \& van Dick, 2014; Offermann, Kennedy Jr., \& Wirtz, 1994): they cognitively anticipate that their teams will develop higher PsyCap because the humble leader profile matches their prototype of a good leader. It is important to note, however, that followers' implicit theories of leadership influence their attitudes and behaviors, in that those leaders who behave in consonance with the leadership prototypes tends to elicit more favorable attitudes and behaviors from their followers (Epitropaki \& Martin, 2004; Junker \& van Dick, 2014). Moreover, the majority of participants (both in the Singaporean and Portuguese samples) indicated prior work experience, which increases the similarity between the experimental and the natural setting and reinforces the subjective experience of being immersed in the situation reported in the vignettes (Aguinis \& Bradley, 2014).

In short, although the experimental vignettes methodology presents several advantages (Aguinis \& Bradley, 2014), additional field experimental studies would be helpful to further support these relationships. Because these reasons, we conducted two additional field studies examining the effects of leader humility on team performance through team PsyCap (Study 2) and serially through team PsyCap and team task allocation effectiveness (Study 3). We also further establish the generalizability of our findings cross-culturally by conducting our Study 3 in China. 
Differently from the Study 1 , these two studies were carried out in real settings and benefit from external validity. We consider that by triangulating the findings of Study 1 with two other studies that do have strong external validity, together our set of studies provide compelling support for our proposed relationships.

\section{STUDY 2 METHODS}

\section{Sample and Procedures}

We recruited 83 teams (middle level; mean team size: 11.24 employees) from local branches (located at the center of Portugal) of 41 organizations (sectors: health care, logistics, consulting, ceramics, retailing, telecommunications, hospitality, banking, automotive industry, and insurance) to participate in this study. Two of the authors contacted the directors of each branch through personal networks (resulting, mainly, from executive training) and explained the purpose of the study. The directors then assisted the authors to randomly select available teams for survey participation.

Teams operated in areas such as marketing, HRM, manufacturing, customer service, logistics, engineering, and finance. We requested the team leaders' permission to deliver a questionnaire to all team members (including the leader). Respondents anonymously reported their perceptions of the leader's humility and team PsyCap, and mailed their responses to the researchers directly. There were a total of 933 team members in the 83 teams, and 308 team members participated, yielding a response rate of $33 \%$. Considering that for 13 of the 83 teams only two participants participated, and to ensure that the respondents accurately reflected their teams (Hinds \& Mortensen, 2005; Langfred, 2005), we restricted the sample to include only teams in which at least three team members had participated ( $n=70$ teams, 282 followers). Followers were an average of 36.02 years old, $52.1 \%$ female, and $36.1 \%$ had a bachelor's degree. Leaders were an average of 43.15 years old, $28.6 \%$ female, and $58.5 \%$ had a bachelor's degree. 
The average leader-follower tenure was 5.78 years. Mean team size was 12.19 employees.

Measures

All variables were measured with a 6-point scale. Respondents were requested to report the extent to which different statements applied to the leader or to the team $(1=$ "the statement does not apply to this leader [this team] at all" to $6=$ "the statement applies completely to this leader [this team]." Leaders rated the team performance. Team members reported the leader's humility and team PsyCap (individual data were then aggregated to the leader/team level; see below).

Leader humility. Because highly humble people tend to underrate their own humility, and those low in humility tend to overrate their own humility (Ou et al., 2015; Tangney, 2009), we followed the leader humility literature and used an other-report measure of leader humility (Ou et al., 2015; Owens \& Hekman, 2016; Owens et al., 2013). Team members rated the leader's humility through nine items (Owens et al., 2013) representing three dimensions: (1) willingness to view oneself accurately, (2) appreciation of others' strengths, and (3) teachability. Sample items include, respectively: (1) “The team leader admits it when (s)he doesn't know how to do something"; (2) "The team leader shows appreciation for the unique contributions of others"; (3) "The team leader is willing to learn from others." In line with a direct consensus composition model (Chan, 1998), these ratings were aggregated to the team level to represent the leader humility score $($ Alpha $=.96)$.

Team PsyCap. Team members reported team PsyCap through the PCQ-12 (Avey et al., 2011) as in Study $1($ Alpha $=.94)$. 
Team performance. Team leaders described team performance with three items (Schaubroeck, Lam, \& Cha, 2007). A sample item is "This team gets its work done very effectively" (Alpha $=.78)$.

\section{Preliminary Analysis}

To ensure that data aggregation is appropriate, we calculated ICC1, ICC2, and $r_{w g(J)}$ for each of the follower-rated constructs (see the above discussion about when data aggregation is appropriate: "Preliminary analysis", Study 1). The ICC1s for leader humility and team PsyCap were .37 and .33 , respectively. The ICC2s for leader humility and team PsyCap were .70 and .66 , respectively. The $r_{w g(J)}$ values for leader humility (average: .85; medium: .94; percentage of values higher than .70: 86\%) and team PsyCap $(.93 ; .96 ; 93 \%)$ represent "strong" and "very strong" inter-rater agreement (LeBreton \& Senter, 2008, p. 836), respectively. Although the ICC2 for team PsyCap is marginally below the recommended cutoff, it does not prevent aggregation. Methodologists (e.g., Bliese, 2000; Chen \& Bliese, 2002) have suggested that a low ICC2 merely reduces statistical power and does not prevent aggregation if aggregation is theoretically justified and $r_{w g(J)}$ is high. We thus proceeded to aggregate our data for these variables.

Prior to hypothesis testing, we conducted CFAs to ensure the discriminant validity among our three key constructs. Given that leader humility and team PsyCap were both theorized to have sub-dimensions, we created three parcels for leader humility and four parcels for team PsyCap to reflect these theoretical underpinnings (Little, Cunningham, Shahar, \& Widaman, 2002). Results indicated that the three-factor structure fit the data well $\left(\chi_{[32]}^{2}=81.86, p<.01 ; \mathrm{RMSEA}=.07 ; \mathrm{CFI}=.98\right)$ and was superior to models in which (1) humility and team PsyCap were set to load on a single factor $\left(\Delta \chi^{2}{ }_{[2]}=76.38\right.$, $p<.01)$ and (2) team PsyCap and team performance were set to load on a single factor 
$\left(\Delta \chi_{[2]}^{2}=22.87, p<.01\right)$. Descriptive statistics and bivariate correlations (correlation between leader humility and team PsyCap based on split samples; see below) are presented in Table 2 .

Insert Table 2 about here

\section{Hypothesis Testing}

To test Hypothesis 1, we conducted a regression analysis and modelled leader humility as the predictor and team PsyCap as the dependent variable. The effect is positive and significant $\left(\beta=.45, p<.001 ; \mathrm{R}^{2}:=.21, p<.01\right)$. To test Hypothesis 2 , we conducted a bias-corrected bootstrap analysis (5,000 samples) with the PROCESS macros developed by Hayes (2013). To reduce the risks of common method variance (CMV), the hypotheses were tested through using different raters to measure humility and team PsyCap. We randomly split the sample in two subsamples and used one subsample to measure leader humility and the other one to measure team PsyCap (Kozlowski, Mak, \& Chao, 2016). The procedure revealed to be appropriate to reduce CMV because the correlation is higher versus lower $(.75, p<.01$, against $.45, p<.01)$ when data come from the same versus different team members. Results suggested that leader humility exerted a significant and positive indirect effect on team performance via increased team PsyCap (indirect effect $=.11, \mathrm{SE}=.07,95 \% \mathrm{CI}=.01$ to .29 ; the direct effect of leader humility on team performance was not significant: effect $=-.05$, $\mathrm{SE}=.10,95 \% \mathrm{CI}=-.25$ to .15 ; effect of team PsyCap on team performance, when leader humility is controlled: $.31, \mathrm{SE}=.13,95 \% \mathrm{CI}=.05$ to .57$)$. A similar pattern emerged when the bias-corrected bootstrap analysis was conducted with 1,000 samples. These results provide convergent support for Hypotheses 1 and 2. 
As these findings are based on cross-sectional data, we performed a robustness check and tested an alternative model. It is plausible to argue that it is easier for leaders to show humility to teams that are already inclined to be hopeful, optimistic, and resilient (i.e., have high team PsyCap). Thus, we tested a model with team PsyCap leading to leader humility and then to team performance; however, the results were not significant (indirect effect $=-.03, \mathrm{SE}=.05,95 \% \mathrm{CI}=-.21$ to .07 ).

\section{Study 2 Discussion}

Although the findings from Study 2 provide support to our hypotheses that humble leaders help foster team PsyCap, which in turn enhanced team performance, it is not without limitations. First, reverse causality is theoretically plausible due to its crosssectional nature even though empirically we ruled it out based on our available data. Second, we were unable to model team allocation effectiveness, a crucial second stage mediator, in this study. Finally, the relatively low team member response rate observed in this study might have influenced the relationships examined. To address each of these limitations, we conducted a multisource, three-wave study with the goals to replicate and extend our findings.

\section{STUDY 3 METHODS}

\section{Participants and Procedures}

We recruited our participants from 10 different Chinese organizations and a variety of functional backgrounds including human resource management, $R \& D$, and sales. To gain organizational entry, one of the authors contacted the senior directors of each firm through personal networks and explained the purpose of the research. The senior directors then assisted us to randomly select available teams for survey participation. We contacted 92 team leaders and 427 followers for our multisource, three-wave. We received data from 83 teams at Time 3. Unfortunately, a number of 
team leaders did not complete the Time 3 performance measure and were dropped from the analyses. We also dropped teams in which fewer than three team members responded, resulting in a final sample size of 53 team leaders (response rate $=57.6 \%$ ) and 203 followers (response rate $=48 \%$ ). Followers were an average of 31.33 years old, $65.5 \%$ male, had an organizational tenure of 5.65 years, and $52.7 \%$ had a bachelor's degree. Leaders were an average of 35.02 years old, 77.4\% male, had an organizational tenure of 9.26 years, and $56.6 \%$ had a bachelor's degree. The average leader-follower tenure was 2.39 years. The average team size was 3.86 .

At Time 1, followers rated their leaders' humility. At Time 2, followers completed measures on PsyCap and task allocation effectiveness; leaders did not provide any data at Time 1 and 2. At Time 3, leaders rated their teams' performance. Each wave was separated by roughly two weeks.

\section{Measures}

Leader humility. Leader humility was measured using the same nine-item scale as in Study 2 (Owens et al., 2013), (Alpha = .92).

Team PsyCap. Team PsyCap was measured using the same 12-item scale as in Studies 1 and 2 (PCQ-12, Avey et al., 2011), (Alpha = .92).

Team task allocation effectiveness. Team task allocation effectiveness was measured using a "team demands-abilities fit" (Abdel-Halim, 1981) five-item individual-level measure aggregated to the team level. Sample items include: "I feel that my team tasks and I are well matched," and "My team tasks give me a chance to do the things I feel I do best” (Alpha $=.88)$.

Team performance. Leader-rated team performance was assessed with the same three-item measure used in Study 2 (Schaubroeck et al., 2007), (Alpha $=.87)$.

\section{Preliminary Analyses}


To ensure data aggregation is appropriate, we calculated ICC1, ICC2, and $r_{w g(J)}$ for each of the follower-rated constructs following the same guidelines used in Studies 1 and 2 (Bliese, 2000; Kozlowski \& Klein, 2000). The ICC1s for leader humility, team PsyCap, and team allocation effectiveness were .30, .21, and .09, respectively. The ICC2s for leader humility, team PsyCap, and team allocation effectiveness were .71, .51 , and .28, respectively. The $r_{w g(J)}$ values for leader humility (average: .97; medium: .98 ; percentage of values higher than .70: 98.79\%), team PsyCap $(.93 ; .97 ; 98.80 \%)$ and team task allocation effectiveness $(.92 ; .96 ; 96.38 \%)$ represent "very strong" interrater agreement (LeBreton \& Senter, 2008). Although the ICC2 for team PsyCap and, mainly, team allocation effectiveness is below the recommended cutoff (one possible reason being the small number of participants in some teams), it does not prevent aggregation (Bliese, 2000; Chen \& Bliese, 2002). For the reasons presented above for Study 2, we thus proceeded to aggregate our data for these variables.

We then conducted a series of CFA analyses to ensure that our focal constructs (i.e., leader humility, team PsyCap, team allocation effectiveness, and team performance) had satisfactory discriminant validity. Results indicated that the fourfactor structure fit the data well $\left(\chi_{[84]}^{2}=159.11, p<.01\right.$; RMSEA $\left.=.06 ; \mathrm{CFI}=.98\right)$, and was superior to models in which (1) team PsyCap and team allocation effectiveness were set to load on a single factor $\left(\Delta \chi_{[3]}^{2}=303.07, p<.01\right)$ and (2) leader humility and team performance were set to load on a single factor $\left(\Delta \chi_{[34]}^{2}=782.14, p<.01\right)$. Descriptive statistics and bivariate corrections are presented in Table 3.

Insert Tables 3 and 4 about here

\section{Tests of Hypotheses}


To test Hypothesis 1, we conducted a regression analysis and positioned leader humility as the predictor and team PsyCap as the dependent variable. Results suggested the effect was positive and significant $\left(\beta=.59, p<.01 ; \mathrm{R}^{2}:=.04, p<.01\right)$. To test Hypothesis 2, we conducted a bias-corrected bootstrap analysis as in Study 2. Considering that data about PsyCap and task allocation effectiveness were collected at the same time (T2) and from the same team members, and to reduce risks of CMV, we randomly split the sample in two subsamples and used each one to measure each variable (Kozlowski et al., 2016). The procedure revealed to be appropriate to reduce CMV because the correlation is higher versus lower $(.61, p<.01$, against $.44, p<.01)$ when data come from the same versus different team members. Results revealed that leader humility exerted a significant and positive indirect effect on team performance via increased team PsyCap (indirect effect $=.07, \mathrm{SE}=.03,95 \% \mathrm{CI}=.01$ to .16 ; the direct effect of leader humility on team performance was not significant: effect $=.26$, $\mathrm{SE}=.25,95 \% \mathrm{CI}=-.19$ to .81$)$. Together with Studies 1 and 2 , these results provide convergent support for Hypotheses 1 and 2.

To test our serial indirect effect model in an integrated fashion, we conducted a bias-corrected bootstrap analysis and modeled leader humility as the independent variable, team PsyCap as the first mediator, team allocation effectiveness as the second mediator, and leader-rated team performance as the dependent variable. Results suggested the serial indirect effect model was significant (indirect effect $=.07, \mathrm{SE}=.03$, $95 \%=.01$ to .16 ) providing support for H3 (see Table 4). Like Study 2, we also provided additional robustness checks by testing alternative sequenced models. For example, since our two mediators were measured at the same point in time, we tested a model wherein we reversed the order of team PsyCap and allocation effectiveness. Results suggested that when the two mediating mechanisms were reversed, the model 
was not significant. Similar to Study 2, we also tested the possibility that heightened team PsyCap may facilitate or encourage more humble forms of leadership or that team PsyCap may be facilitated by good performance, but neither of these models were significant (all robustness checks are reported in Table 4). Together, these analyses provide strong support for Hypothesis 3.

\section{Study 3 Discussion}

In this study, we tested our full theorized model, replicating the findings of Study 2 in a different culture with a different set of organizations, and extending beyond Study 2 by modeling team task allocation effectiveness. This study also has strengths that address some of the design weaknesses of Study 2; namely, in Study 3 we sought to increase the rigor of our findings by employing multi-stage survey design, which helps to reduce CMV concerns. In addition, our team member response rate was much high in Study 3, compared to Study 2. In summary, by triangulating the experimental findings of Study 1, the initial field-study findings of Study 2, and the more robust findings of Study 3, we sought to present strong overall support for our proposed theoretical model.

\section{GENERAL DISCUSSION}

The study corroborates extant literature suggesting that leader humility is relevant for team performance (e.g., Owens \& Hekman, 2016) and that the virtue of humility, contrarily to what Hunter (2000) argued about the "death of character", is not “dead" (Wright \& Goodstein, 2007). Our findings support Wright and Goodstein (2007, p. 929) who consider virtues as "possible determinants of both individual betterment and organizational efficiency and performance." The study also corroborates that humble leaders do not affect performance directly; rather, they adopt behaviors and create conditions for employees and teams to perform well. In this article, we apply social information processing theory and humble leadership grounded theory to 
understand how leader humility influences team performance. Using samples from organizations in Singapore, Portugal, and China, we empirically demonstrate that leader humility builds team strengths in the form of team PsyCap, which influences the team process of task allocation effectiveness to improve team performance.

This article makes several important contributions to the literature. First, this research adds to the growing leader humility literature and expands the nomological net by examining team PsyCap as a result of leader humility. The classic virtue of humility is recognized as a foundation for building other strengths, and this research empirically confirms that team PsyCap is among the strengths developed within the followers of humble leaders. This finding is particularly relevant to the positive organizational behavior literature, as it opens up the notion of PsyCap development to include leader behaviors like openness to ideas and appreciation of others' strengths, which goes beyond the traditional development channels of authentic leadership and PsyCap contagion (Luthans et al., 2015). While recent research has shown that humility is a strength in that it can compensate for lower intelligence and is a strong predictor of adaptive performance improvement (Owens et al., 2013), the current research is among the first attempts to show that humility can help foster virtues, strength, or excellence in others, who are the receivers of humble behaviors. Building off of recent research which has documented the influence of leader humility on team humility (Owens \& Hekman, 2016), we show that leader humility can foster collective character strengths in the teams they lead.

Second, this study adds invaluable understanding to the leader humility literature as it demonstrates the value of these behaviors across different cultures (in Singapore, Portugal, and China). Leader humility is a nontraditional leadership approach with benefits that could be mitigated by a culture high in power distance, masculinity, or 
uncertainty avoidance. Yet this cross-cultural examination of the benefits of leader humility indicates that it corresponds with higher team PsyCap and/or team performance in three different cultures with stark differences in the cultural values of power distance, masculinity, long-term orientation, and uncertainty avoidance (Hofstede, 2016a, 2016b, 2016c; Hofstede et al., 2010). While Eastern cultures have long embraced the concept of humility and therefore generally may be more receptive to this leadership approach relative to those in Western cultures (Morris et al., 2004; Oc et al., 2015), our findings show that the impact of leader humility on followers is similar in Singapore, China, and Portugal, which begins to establish the generalizability of leader humility influence across cultures.

However, two main findings recommend complementing such a cross-cultural perspective with an indigenous one (Gelfand, Erez, \& Aycan, 2007). First, in Study 1, although both Singaporean and Portuguese participants reacted similarly in the humble condition, they reacted differently to the control condition: Singaporean versus the Portuguese participants react more negatively to a leader characterized by low humility. It is possible that in contexts where the value of humility is more versus less strongly endorsed (Mittal \& Dorfman, 2012; Oc et al., 2015), individuals are more versus less sensitive to leaders who do not show humility. Future studies should explore the issue. Second, comparing the ratings of leader humility across Studies 2 and 3, leader humility ratings in China were an average of one point higher than leader humility ratings in Portugal, suggesting perhaps an increased prevalence of humble leadership in China. Authors like Ou et al. (2015), Peterson and Seligman (2004), and Vera and RodriguezLopez (2004) argue that Asian cultures have a stronger natural inclination toward humility. Mittal and Dorfman (2012) also show that humility is considered more important for effective leadership in the Confucian Asia and Southern Asia clusters (see 
Oc et al., 2015) than in the Latin Europe cluster. Considering the findings of Study 1, a complementary explanation is that individuals from those Asian contexts, by espousing humility as an important social value, are especially sensitive to low levels of humility of their leaders.

Third, we also address the scarcity of studies on PsyCap at the collective level and enrich understanding about the team processes that team PsyCap can catalyze. Our research demonstrates how team PsyCap improves team effectiveness by maximizing the use of the team member strengths by matching tasks with members' unique expertise and abilities. This research illuminates how team PsyCap fosters task work processes that are more proximal to team performance, which represents a novel contribution in the literature. The current findings help to contribute to PsyCap because they extend beyond cognitive, motivational, and attitudinal correlates to anchor PsyCap to behavioral task work processes, such as task allocation effectiveness. While we focused on task allocation effectiveness, future research in this area should consider additional team processes that might be influenced by leader humility and team PsyCap, such as decision making and conflict management.

\section{Limitations and Future Research}

Although the findings of our experimental study, our model replication and extension across cultures, and our three-wave Study 3 design represent some important methodological strengths, there are also some limitations that should be considered when interpreting the results of our studies. First, such as discussed above, although our experimental study allows more rigorously confirming the causal direction of our proposed model, (a) it was carried out through scenarios, instead of with observed real behaviors, (b) only the link between leader humility and team PsyCap (H1) was studied, and (c) it is possible that the findings represent, at least partially, the effects of 
participants' implicit theory of leadership. It is noteworthy however that such a possible "implicit theorized" relationship is corroborated by the findings of the two field studies carried out in two different contexts.

Second, Studies 2 and 3 may suffer from possible nonresponse bias, in that the teams or leaders that did not respond may be qualitatively different from those that responded. Moreover, in those two studies, we used a survey design rather than a field experiment in testing our models, which limits the causal inferences we can make. We believe that relative to Study 2, our multiwave Study 3 helps to more clearly demonstrate the causal relationships among team humility, team PsyCap, task allocation effectiveness, and team performance. In addition, we provide a robustness check by reporting nonsignificant indirect effects of alternately ordered models. Although each study has its own weaknesses (e.g., Study 3's mediators were both reported at T2, Study 1 was modest in external validity), we believe the combination of these three studies provides consistent and convergent support for our theoretical model.

Third, as we rely on leader-rated performance in Studies 2 and 3, humble (not humble) leaders might positively inflate (deflate) performance ratings for followers they are apt to view (less) appreciatively. However, we note that in past humility research both objective and subjective performance measures have shown positive correlations with humility (Owens \& Hekman, 2016) and that in Study 3 we also triangulated our results with team member ratings of performance and found similar effects (i.e., all hypothesis testing yielded equivalent results when using aggregate team-member rated performance). In addition, humble leaders are also inclined to not conceal or gloss over mistakes and emphasize the value of growth (Owens \& Hekman, 2012). This inclination may translate to giving accurate assessments of followers' and helping them grow by 
pointing out areas for improvement. That being said, we encourage future research to replicate our findings with objective performance measures.

Fourth, across the three studies we show that leader humility has a similar influence on work teams in Singapore, Portugal, and China. However, no cultural variables were directly measured or tested in either study. While the supported hypotheses speak both to the generalizability of the value of leader humility and the strength of the model being tested, we encourage future research to explore cultural differences in the demonstration and effectiveness of leader humility by directly assessing the employee cultural values. We also encourage future studies to identify, in a clearer way, the influence of both local/indigenous and cross-cultural factors on the hypothesized relationships.

Furthermore, though we believe the proposed indirect effects of team PsyCap and task allocation effectiveness are important, we believe there may be other mechanisms through which leader humility shapes or facilitates team effectiveness. In light of the principle of equifinality (Morgeson, DeRue, \& Karam, 2010), we acknowledge the possibility that leader humility may influence team performance through other emergent states and processes (i.e., learning goal orientation, team members participation in decision making, mutual trust, psychological safety and speaking up, prevalent conflict management style within the team, collective organizational citizenship behavior, team potency) and encourage future research to theorize and explore other team-level variables that mediate the impact of leader humility on team effectiveness. For example, it is possible that humble leaders encourage a team climate characterized by speaking up and integrative conflict management behaviors, thus increasing the teams' confidence in being able to achieve its most challenging tasks, which in turn increases team effectiveness. 


\section{Practical Implications}

Our findings have practical value for leadership and team effectiveness. Specifically, training leaders that taking a humble leadership approach with their teams can help facilitate a positive state of development for team members. Leaders might consider enacting humble leadership behaviors, such as modeling teachability, highlighting followers' strengths, diverting praise to followers, and mentoring/emphasizing followers' developmental journeys (Owens \& Hekman, 2012) in order to augment team PsyCap. This research also suggests that a leader's failure to display the behaviors of humility may inhibit the development of the team PsyCap dimensions. In addition, if organizations want to foster task allocation effectiveness within their work teams, leaders should show humility by spotlighting specific team member strengths, which would socially reinforce awareness, efficacy, and eventual utilization of these strengths.

\section{Conclusion}

As noted by Weick (2001, p. 93), increased "unpredictability and unknowability" within modern organizations requires leaders to have "more humility and less hubris." Our paper suggests that leader humility helps build adaptive strengths within followers (team PsyCap) and make fuller use of their human capital (task allocation effectiveness), which may help them deal with this increased unpredictability within the workplace. Across three studies and three cultures (in Singapore, Portugal, and China), we find consistent support for the positive impact of leader humility on team emergent states, team processes, and team effectiveness. We hope these findings spur further research on the role and relevance of the historic strength of humility within contemporary contexts. 


\section{REFERENCES}

Abdel-Halim, A. A. 1981) Effects of role stress-job design-technology interaction on employee work satisfaction. Academy of Management Journal, 24: 260-273.

Aguinis, H., \& Bradley, K. 2014. Best practice recommendations for designing and implementing experimental vignette methodology studies. Organizational Research Methods. doi:10.1177/1094428114547952

Aristotle. 1999. Nicomachean ethics. Kitchener, Ontario, Canada: Batoche Books.

Avey, J. B., Reichard, R. J., Luthans, F., \& Mhatre, K. H. 2011. Meta-analysis of the impact of positive psychological capital on employee attitudes, behaviors, and performance. Human Resource Development Quarterly, 22: 127-152.

Awtry, M. 2007. River of life: How to live in the flow. Bloomington, IN: AuthorHouse.

Bandura, A. 1997. Self-efficacy: The exercise of control. New York, NY: Freeman.

Bandura, A., \& Locke, E. A. 2003. Negative self-efficacy and goal effects revisited. Journal of Applied Psychology, 88: 87-99.

Basford, T. E., Offermann, L. R., \& Behrend, T. S. 2014. "Please accept my sincerest apologies:” Examining follower reactions to leader apology. Journal of Business Ethics, 119: 99-117.

Bliese, P. D. 2000. Within-group agreement, non-independence, and reliability: Implications for data aggregation and analysis. In K. J. Klein \& S. W. Kozlowski (Eds.), Multilevel theory, research, and methods in organizations: 349-381. San Francisco, CA: Jossey-Bass.

Bloomfield, P. 2000. Virtue epistemology and the epistemology of virtue. Philosophical and Phenomenological Research, 60: 23-43.

Brandon, D.P., \& Hollingshead, A.B. 2004. Transactive memory systems in organizations: Matching tasks, expertise, and people. Organization Science, 15: 633-644. 
Brislin, R. W. 1970. Back-translation for cross-cultural research. Journal of Crosscultural Psychology, 1: 185-216.

Burke, C. S., Stagl, K. C., Klein, C., Goodwin, G. F., Salas, E., \& Halpin, S. M. 2006. What type of leadership behaviors are functional in teams? A meta-analysis. The Leadership Quarterly, 17: 288-307.

Cameron, K., \& Spreitzer, G. (Eds.). 2012. Handbook of positive organizational scholarship. New York, NY: Oxford University Press.

Cameron, K., \& Winn, B. 201. Virtuousness in Organizations. In K. S. Cameron \& G. Spreitzer (Eds.), The Oxford handbook of positive organizational scholarship: 231-243. Oxford, England: Oxford University Press.

Chan, D. 1998. Functional relations among constructs in the same content domain at different levels of analysis: A typology of composition models. Journal of Applied Psychology, 83: 234-246.

Chen, G., \& Bliese, P. D. 2002. The role of different levels of leadership in predicting self- and collective efficacy: Evidence for discontinuity. Journal of Applied Psychology, 87: 549-556.

Clapp-Smith, R., Vogelgesang, G. R., \& Avey, J. B. 2009. Authentic leadership and positive psychological capital: the mediating role of trust at the group level of analysis. Journal of Leadership \& Organizational Studies, 15: 227-240.

Colquitt, J. A., \& Zapata-Phelan, C. P. 2007. Trends in theory building and theory testing: A five-decade study of the Academy of Management Journal. Academy of Management Journal, 50: 1281-1303.

Colvin, C. R., Block, J., \& Funder, D. C. 1995. Overly positive self-evaluations and personality: negative implications for mental health. Journal of Personality and Social Psychology, 68: 1152-1162. 
Dawkins, S., Martin, A., Scott, J., \& Sanderson, K. 2015. Advancing the conceptualization and measurement of Psychological Capital as a collective construct. Human Relations, 68: 925-949.

The Economist. (2013). Davos Man and his defects. Retrieved from http://www.economist.com/news/business/21570684-global-leadership-industryneeds-re-engineering-davos-man-and-his-defects

Epitropaki, O., \& Martin, R. 2004. Implicit leadership theories in applied settings: Factor structure, generalizability, and stability over time. Journal of Applied Psychology, 89: 293-310.

Felfe, J., \& Schyns, B. 2006. Personality and the perception of transformational leadership: The impact of extraversion, neuroticism, personal need for structure, and occupational self-efficacy. Journal of Applied Social Psychology, 36: 708739.

Gelfand, M. J., Erez, M., \& Aycan, Z. 2007. Cross-cultural organizational behavior. Annual Review of Psychology, 58: 479-514.

Grenberg, J. M. 2005. Kant and the ethics of humility: A story of dependence, corruption and virtue. Cambridge, England: Cambridge University Press.

Grant, A. M., Gino, F., \& Hoffman, D. A. 2011. Reversing the extraverted leadership advantage: The role of employee proactivity. Academy of Management Journal, 54: $528-550$.

Haar, J. M., Roche, M., \& Luthans, F. 2014. Do leaders' psychological capital and engagement influence follower teams or vice versa? Academy of Management, Annual Meeting Proceedings, 1: 11058-11058.

Hayes, A. F. 2013. Introduction to mediation, moderation, and conditional process analysis. New York, NY: Guilford Press. 
Heled, E., Somech, A., \& Waters, L. 2016. Psychological capital as a team phenomenon: Mediating the relationship between learning climate and outcomes at the individual and team levels. The Journal of Positive Psychology, 11: 303314.

Hinds, P. J., \& Mortensen, M. 2005. Understanding conflict in geographically distributed teams: The moderating effects of shared identity, shared context, and spontaneous communication. Organization Science, 16: 290-307.

Hofstede, G. 2016a. The Hofstede centre. Retrieved from http://geerthofstede.com/portugal.html

Hofstede, G. 2016b. The Hofstede centre. Retrieved from http://geerthofstede.com/china.html

Hofstede, G. 2016c. The Hofstede centre. Retrieved from https://geerthofstede.com/singapore.html

Hofstede, G., Hofstede, G. J., \& Zinkov, M. 2010. Cultures and organizations: Software of the mind. New York, NY: McGraw-Hill.

Hunter, J. W. 2000. The death of character: Moral education in an age without good or evil. New York. NY: Basic Books.

Ilgen, D. R., Hollenbeck, J. R., Johnson, M., \& Jundt, D. 2005. Teams in organizations: From input-process-output models to IMOI models. Annual Review of Psychology, 56: 517-543.

Junker, N. M., \& van Dick, R. 2014. Implicit theories in organizational settings: A systematic review and research agenda of implicit leadership and followership theories. The Leadership Quarterly, 25: 1154-1173.

Kesebir, P. 2014. A quiet ego quiets death anxiety: Humility as an existential anxiety buffer. Journal of Personality and Social Psychology, 106: 610-623. 
Kozlowski, S. W., \& Klein, K. J. 2000. A multilevel approach to theory and research in organizations: Contextual, temporal, and emergent processes. In K. J. Klein \& S. W. J. Kozlowski (Eds.), Multilevel theory, research and methods in organizations: Foundations, extensions, and new directions: 3-90. San Francisco, CA: Jossey-Bass.

Kozlowski, S. W. J., Mak, S., \& Chao, G. T. 2016. Team-centric leadership: An integrative review. Annual Review of Organizational Psychology and Organizational Behavior, 3: 21-54.

Krieger, A. 2002. Arguing the 'against' position: new urbanism as a means of building and rebuilding our cities. In T. W. Bressi (Ed.), The Seaside debates: A critique of the new urbanism: 51-58. New York, NY: Rizzoli.

Langfred, C. W. 2005. Autonomy and performance in teams: The multilevel moderating effect of task interdependence. Journal of Management, 31: 513-529.

LeBreton, J. M., \& Senter, J. L. 2008. Answers to 20 questions about interrater reliability and interrater agreement. Organizational Research Methods, 11: 815852.

Lengnick-Hall, C. A., Beck, T. E., \& Lengnick-Hall, M. L. 2011. Developing a capacity for organizational resilience through strategic human resource management. Human Resource Management Review, 21: 243-255.

Lewis, K. 2003. Measuring transactive memory systems in the field: Scale development and validation. Journal of Applied Psychology, 88: 587-604.

Little, T. D., Cunningham, W. A., Shahar, G., \& Widaman, K. F. 2002. To parcel or not to parcel: Exploring the question, weighing the merits. Structural Equation Modeling, 9: 151-173. 
Lubatkin, M. H., Simsek, Z., Ling, Y., \& Veiga, J. F. 2006. Ambidexterity and performance in small- to medium-sized firms: The pivotal role of top management team behavioral integration. Journal of Management, 32: 646-672.

Luthans, F., \& Youssef, C. 2004. Human, social, and now positive psychological capital management: Investing in people for competitive advantage. Organizational Dynamics, 33: 143-160.

Luthans, F., Youssef-Morgan, C., \& Avolio, B. J. 2015. Psychological capital and beyond. New York, NY: Oxford University Press.

Luthans, F., Avolio, B. J., Avey, J. B., \& Norman, S. M. 2007. Positive psychological capital: Measurement and relationship with performance and satisfaction. Personnel Psychology, 60: 541-572.

Marks, M. A., Mathieu, J. E., \& Zaccaro, S. J. 2001. A temporally based framework and taxonomy of team processes. Academy of Management Review, 26: 356-376.

Mathe-Soulek, K., Scott-Halsell, S., Kim, S., \& Krawczyk, M. 2014. Psychological capital in the quick service restaurant industry: A study of unit-level performance. Journal of Hospitality \& Tourism Research. doi:1096348014550923

Mathieu, J. E., Heffner, T. S., Goodwin, G. F., Sales, E., \& Cannon-Bowers, J. A. 2000. The influence of shared mental models on team process and performance. Journal of Applied Psychology, 85: 273-283.

Mittal, R., \& Dorfman, P. W. 2012. Servant leadership across cultures. Journal of World Business, 47: 555-570.

Moreland, R. L. 2006. Transactive memory: Learning who knows what in work groups and organizations. In J. M. Levine \& R. R. Moreland (Eds.), Small groups: Key readings in social psychology: 327-346. New York, NY: Psychology Press. 
Morgeson, F. P., DeRue, D. S., \& Karam, E. P. 2010. Leadership in teams: A functional approach to understanding leadership structures and processes. Journal of Management, 36: 5-39.

Morris, J. A., Brotheridge, C. M., \& Urbanski, J. C. 2005. Bringing humility to leadership: Antecedents and consequences of leader humility. Human Relations, 58: $1323-1350$.

Oc, B., Basshur, M. R., Daniels, M. A., Greguras, G. J., \& Diefendorff, J. M. 2015. Leader humility in Singapore. The Leadership Quarterly, 26: 68-80.

Offermann, L. R., Kennedy Jr., J. K., \& Wirtz, P. W. 1994. Implicit leadership theories: Content, structure, and generalizability. The Leadership Quarterly, 5: 43-58.

Ou, A. Y., Tsui, A. S., Kinicki, A. J., Wladman, D. A., Xiao, Z., \& Song, L. J. (2014). Humble Chief Executive Officers' connections to top management team integration and middle managers' responses. Administrative Science Quarterly, 59: $34-72$.

Ou, A. Y., Waldman, D. A., \& Peterson, S. J. 2015. Do humble CEOs matter? An examination of CEO humility and firm outcomes. Journal of Management. doi:10.1177/0149206315604187

Owens, B. P., Johnson, M. D., \& Mitchell, T. R. 2013. Expressed humility in organizations: Implications for performance, teams, and leadership. Organization Science, 24: 1517-1538.

Owens, B. P., \& Hekman, D. R. 2016. How does leader humility influence team performance? Exploring the mechanisms of contagion and collective promotion focus. Academy of Management Journal, 58: 1088-1111. 
Owens, B. P., \& Hekman, D. R. 2012. Modeling how to grow: An inductive examination of humble leader behaviors, contingencies, and outcomes. Academy of Management Journal, 55: 787-818.

Owens, B. P., Wallace, A. S., \& Waldman, D. A. 2015. Leader narcissism and follower outcomes: The counterbalancing effect of leader humility. Journal of Applied Psychology, 100: 1203.

Peterson, C., \& Seligman, M. E. P. 2004. Character strengths and virtues: A handbook and classification. New York, NY: Oxford University Press.

Roberts, R. C. \& Wood, W. J. 2003. Humility and epistemic goods. Intellectual Virtue, 1: 257-298.

Salancik, G. R., \& Pfeffer, J. 1978. A social information processing approach to job attitudes and task design. Administrative Science Quarterly, 23: 224-253.

Schaubroeck, J., Lam, S. S. K., \& Cha, S. E. 2007. Embracing transformational leadership: Team values and the impact of leader behavior on team performance. Journal of Applied Psychology, 92: 1020-1030.

Stichter, M. 2007. Ethical expertise: The skill model of virtue. Ethical Theory and Moral Practice, 10: 183-194.

Stichter, M. 2011. Virtues, skills, and right action. Ethical Theory and Moral Practice, 14: 73-86.

Tangney, J. P. 2009. Humility. In S. Lopez \& C. Snyder (Eds.), Oxford Handbook of Positive Psychology (pp. 483-490). New York, NY: Oxford University Press.

Tiedens, L. Z., Unzueta, M. M., \& Young, M. J. 2007. The desire for hierarchy?: The motivated perception of dominance complementarity in task partners. Journal of Personality and Social Psychology, 93: 402-414. 
Van Dierendonck, D. 2011. Servant leadership: A review and synthesis. Journal of Management, 37: 1228-1261.

van Dierendonck, D., Stamn, D., Boersma, P., de Windt, N., \& Alkema, J. 2014. Same difference? Exploring the differential mechanisms linking servant leadership and transformational leadership to follower outcomes. The Leadership Quarterly, 25: $544-562$.

Van Doorn, E. A., Heerdink, M. W., \& Van Kleef, G. A. 2012. Emotion and the construal of social situations: Inferences of cooperation versus competition from expressions of anger, happiness, and disappointment. Cognition \& Emotion, 26: $442-461$.

Van Kleef, G. A., De Dreu, C. K., \& Manstead, A. S. 2004. The interpersonal effects of emotions in negotiations: a motivated information processing approach. Journal of Personality and Social Psychology, 87: 510-528.

Van Kleef, G. A., De Dreu, C. K., \& Manstead, A. S. 2006. Supplication and appeasement in conflict and negotiation: The interpersonal effects of disappointment, worry, guilt, and regret. Journal of Personality and Social Psychology, 91: 124-142.

Vanno, V., Kaemkate, W., \& Wongwanich, S. 2015. Effect of group-level positive psychological capital on group effectiveness of Thai students. Procedia-Social and Behavioral Sciences, 171: 1309-1314.

Vera, D., \& Rodriguez-Lopez, A. 2004. Strategic virtues: Humility as a source of competitive advantage. Organizational Dynamics, 33: 393-408.

Wang, J., Wang, G. G., Ruona, W. E. A., \& Rojewski, J. W. 2005. Confucian values and the implications for international HRD. Human Resource Development International, 8: 311-326. 
Watts, R. E. 2011. Embracing both a constructivist counseling approach and a specific religious tradition: Is it a leap of faith? Counseling and Values, 56: 3-9.

Weick, K. E. 2001. Leadership as the legitimation of doubt. In W. Bennis, G. Spreitzer, \& T. Cummings (Eds.), The future of leadership: Today's top leadership thinkers speak to tomorrow's leaders: 91-102. San Francisco, CA: Jossey-Bass.

West, B. J., Patera, J. L., \& Carsten, M. K. 2009. Team level positivity: investigating positive psychological capacities and team level outcomes. Journal of Organizational Behavior, 30: 249-267.

Wright, T., \& Goodstein, J. 2007. Character is not "Dead" in management research: A review of individual character and organizational-level virtue. Journal of Management, 33: 928-958.

Yaffe, T., \& Kark, R. 2011. Leading by example: The case of leader OCB. Journal of Applied Psychology, 96: 806-826.

Youssef, C., \& Luthans, F. 2012. Psychological capital: Meaning, findings and future directions. In K. S. Cameron \& G. Spreitzer (Eds.), The Oxford handbook of positive organizational scholarship: 17-27. Oxford, England: Oxford University Press. 


\section{Footnote}

1. Copyright (C) 2007 Psychological Capital Questionnaire (PCQ) by Fred L. Luthans, Ph.D., Bruce J. Avolio, Ph.D., \& James A. Avey, Ph.D. All rights reserved in all medium. Distributed by Mind Garden, Inc. The scale of the instrument was modified, by the customer, from the original. 
Table 1

\section{Model Summary}

\begin{tabular}{|c|c|c|c|c|}
\hline $\begin{array}{l}\text { Study } 1 \\
\text { Method (experimental): two samples, one collected in } \\
\text { Singapore }(n=23 \text { teams), other in Portugal }(n=74)\end{array}$ & Leader Humility $\rightarrow$ & $\begin{array}{l}\text { (Expectations of) Team } \\
\text { PsyCap }\end{array}$ & & \\
\hline $\begin{array}{l}\text { Study } 2 \\
\text { Method (multisource cross-sectional): } 70 \text { teams } \\
\text { collected in Portugal; data collected at a single moment; } \\
\text { the three variables measured through data from team } \\
\text { leader (team performance) and different team members } \\
\text { (humility; PsyCap) }\end{array}$ & Leader Humility $\rightarrow$ & Team PsyCap & $\rightarrow$ & Team Performance \\
\hline $\begin{array}{l}\text { Study } 3 \\
\text { Method (three-wave, multisource): } 53 \text { teams collected } \\
\text { in China; data collected at three moments (T1: } \\
\text { humility, data from team members; T2, PsyCap, data } \\
\text { from team members; T3, team performance, data from } \\
\text { team leader) }\end{array}$ & Leader Humility $\rightarrow$ & Team PsyCap $\rightarrow$ & $\begin{array}{l}\text { Team Task Allocation } \\
\text { Effectiveness } \rightarrow\end{array}$ & Team Performance \\
\hline Team Construct Type & Team Input & $\begin{array}{l}\text { Emergent State: Team } \\
\text { Shared Motivational State }\end{array}$ & $\begin{array}{l}\text { Task work: Maximizing } \\
\text { Team Human Capital }\end{array}$ & Team Output \\
\hline Aggregation Logic & $\begin{array}{l}\text { Composition: Direct } \\
\text { Consensus }\end{array}$ & $\begin{array}{l}\text { Composition: Referent-Shift } \\
\text { Consensus }\end{array}$ & Composition: Additive & Team leader rating \\
\hline Definition & $\begin{array}{l}\text { Leaders modeling to followers } \\
\text { the behaviors of admitting } \\
\text { mistakes and limitations, } \\
\text { spotlighting follower } \\
\text { strengths, deflecting praise to } \\
\text { others, and being teachable- } \\
\text { open to new ideas, advice, and } \\
\text { feedback. }\end{array}$ & $\begin{array}{l}\text { Team PsyCap "relates to } \\
\text { agreement among team } \\
\text { members in regard to the } \\
\text { team's shared (team- } \\
\text { referent) PsyCap perception } \\
\text { — characterized by hope, } \\
\text { efficacy, optimism and } \\
\text { resilience" (Dawkins et al., } \\
\text { 2015, p. 936) }\end{array}$ & $\begin{array}{l}\text { Team member perceptions } \\
\text { that task/roles are allocated } \\
\text { according to member } \\
\text { strengths (theoretically } \\
\text { centered on the concept of } \\
\text { demands-abilities fit). }\end{array}$ & \\
\hline
\end{tabular}


Table 2

Study 2 Descriptive Statistics and Bivariate Correlations

\begin{tabular}{llllll}
\hline Variables & $M$ & $S D$ & 1 & 2 & 3 \\
\hline 1. Leader humility (follower-reported) & 4.08 & .88 & $(.96)$ & & \\
2. Team psychological capital (follower-reported) & 3.96 & .70 & $.45^{* *}$ & $(.94)$ & \\
3. Team performance (leader-reported) & 5.17 & .69 & .08 & $.28^{* *}$ & $(.78)$ \\
\hline
\end{tabular}

$N=70$ teams. Within brackets, in the diagonal: Cronbach Alphas.

${ }^{*} p<.05$

$* * p<.01$ 
Table 3

Study 3 Descriptive Statistics and Bivariate Correlations

\begin{tabular}{|c|c|c|c|c|c|c|}
\hline Variables & $M$ & $S D$ & 1 & 2 & 3 & 4 \\
\hline 1. Leader humility (follower-reported; T1) & 5.21 & .47 & $(.92)$ & & & \\
\hline 2. Team PsyCap (follower-reported; T2) & 4.02 & .41 & $.41 * *$ & $(.92)$ & & \\
\hline 3. Task allocation effectiveness (follower- & 4.15 & .39 & .18 & $.44 * *$ & $(.88)$ & \\
\hline \multicolumn{7}{|l|}{ reported; T2) } \\
\hline 4. Team performance (leader-reported; T3) & 3.54 & .46 & $.31^{*}$ & $25^{\dagger}$ & $.40 * *$ & $(.87)$ \\
\hline \multicolumn{7}{|c|}{$N=53$ teams. Within brackets, in the diagonal: Cronbach Alphas. } \\
\hline \multicolumn{7}{|l|}{${ }^{\dagger} p<.10$} \\
\hline \multicolumn{7}{|l|}{$* p<.05$} \\
\hline$* * p<.01$ & & & & & & \\
\hline
\end{tabular}

